\title{
Beatmung bei COPD: von der Präklinik bis zur außerklinischen Beatmung. Eine Übersicht des Arbeitskreises für Beatmung und Intensivmedizin der österreichischen Gesellschaft für Pneumologie
}

\author{
Eveline Kink · Lorenz Erler · Wilfried Fritz · Georg-Christian Funk · Martin Gäbler · Florian Krenn • \\ Gernot Kühteubl · Otmar Schindler · Theodor Wanke
}

Online publiziert: 20. Mai 2019

(C) Der/die Autor(en) 2019

Zusammenfassung Das Positionspapier wurde vom Arbeitskreis Beatmung und Intensivmedizin der Österreichischen Gesellschaft für Pneumologie mit dem Ziel erstellt, die Besonderheiten der mechanischen Beatmung bei Patienten mit chronisch obstruktiven Lungenerkrankungen (COPD) zusammenzufassen. Von der akuten respiratorischen Insuffizienz bis zur außerklinischen Beatmung werden die pathophysiologischen Grundlagen sowie die wesentlichen Unterschiede zur Beatmung bei anderer Indikation dargestellt.

Dr. E. Kink, MBA · Dr. O. Schindler

Abteilung für Innere Medizin und Pneumologie, LKH Graz II, Standort Enzenbach, Hörgas 30, 8112 Gratwein-Straßengel, Österreich

Dr. L. Erler · Dr. F. Krenn

Abteilung für Lungenkrankheiten, Leoben, Österreich

\section{Dr. W. Fritz}

Klinische Abteilung für Lungenkrankheiten,

Universitätsklinikum für Innere Medizin, LKH.-Univ.

Klinikum Graz, Graz, Österreich

Prim. PD Dr. G.-C. Funk $(\bowtie)$

2. Medizinische Abteilung mit Pneumologie,

Wilhelminenspital, Wien, Österreich

georg-christian.funk@meduniwien.ac.at

Univ. Lekt. Dr. M. Gäbler, MSc

Institut für Präventiv- und Angewandte Sportmedizin, Universitätsklinikum Krems, Karl Landsteiner

Privatuniversität für Gesundheitswissenschaften,

Mitterweg 10, 3500 Krems an der Donau, Österreich

Martin.Gaebler@kl.ac.at

Dr. G. Kühteubl

Lk Hochegg, Hochegg, Österreich

Univ. Doz. Dr. T. Wanke

Görgengasse 23/8/15, 1190 Wien, Österreich
Schlüsselwörter Chronisch obstruktive Lungenerkrankung (COPD) • Respiratorische Insuffizienz • Mechanische Beatmung · Weaning · Außerklinische Beatmung

Summary This paper was created by the Austrian Society of Pneumology (Working group Ventilation and Intensive Care) to summarize the specific characteristics of mechanical ventilation in patients presenting with chronic obstructive pulmonary disease (COPD). The main differences in pathophysiology and mechanical ventilation are shown, including acute respiratory failure and out-of-hospital mechanical ventilation.

Keywords Chronic obstructive pulmonary disease (COPD) · Respiratory failure · Mechanical ventilation · Weaning · Out-of-hospital mechanical ventilation

\section{Pathophysiologischer Hintergrund}

Bei COPD kommt es aufgrund einer exspiratorischen Atemflusslimitation zum Phänomen der gefangenen Luft (Airtrapping) mit Ausbildung eines intrinsischen positiven endexspiratorischen Drucks (PEEPi). Der PEEPi muss zu Beginn jeder Einatembemühung überwunden werden und trägt zusammen mit der erhöhten Resistance und erniedrigten Thoraxwandcompliance zur vermehrten inspiratorischen Atemarbeit sowie zur gesteigerten $\mathrm{CO}_{2}$-Bildung bei. Der wachsende Totraumanteil in der überblähten Lunge bedingt eine zunehmende Hyperkapnie. Die insuffizient arbeitende Atemmuskulatur ist mit der erhöhten Atemarbeit überfordert, sodass ein Atempumpversagen resultiert (Abb. 1).

Unter Anwendung einer positiven Druckbeatmung wird eine Entblähung ermöglicht, die inspiratorische 
Abb. 1 Mechanismen der respiratorischen Insuffizienz (schwarz) bei COPD und Möglichkeiten der therapeutischen Beeinflussung (grün). Respiratorisches Coaching Sicherstellen einer langen Exspirationszeit, $P E E P i$ intrinsischer PEEP, PEEPe externer PEEP, $V T$ Tidalvolumen

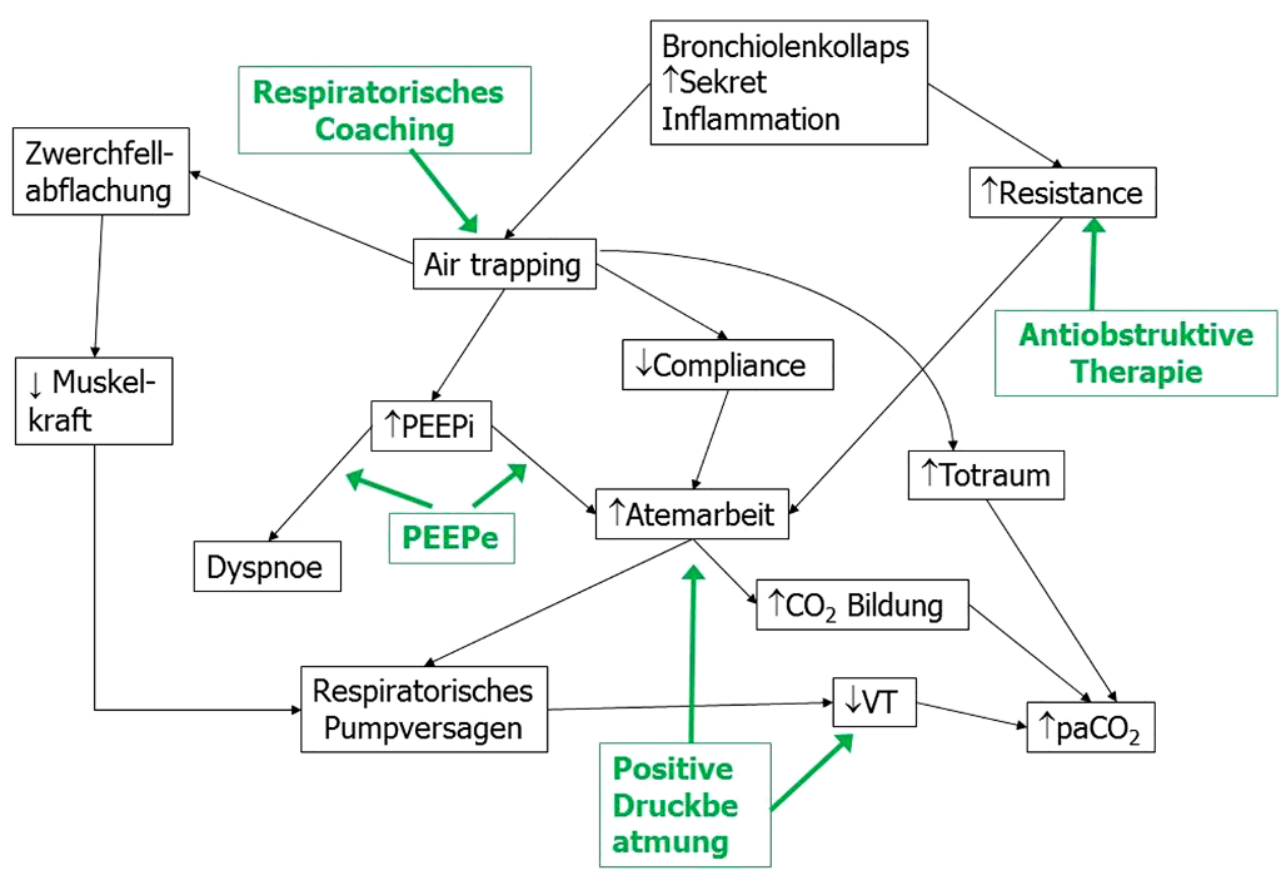

Atemarbeit und folglich auch die Hyperkapnie reduziert. Die Verwendung von externem PEEP (PEEPe) soll außerdem zur Schienung der Atemwege beitragen $[1,2]$.

\section{Empfohlene Beatmungsformen und Einstellparameter bei COPD}

\section{Externer PEEP (PEEPe)}

Der PEEPe kann bei Patienten mit COPD die flussunwirksame inspiratorische Atemarbeit verringern, dadurch wird das Triggern bei assistierter Beatmung erleichtert. Auch die exspiratorische Atemmechanik kann durch eine Schienung der Atemwege verbessert werden. Ein zu hoher PEEPe jedoch trägt zur Überblähung und somit zu erhöhter Atemarbeit bei. Der optimale PEEPe beträgt ca. $80 \%$ des intrinsischen PEEP (PEEPi). Da eine Messung des intrinsischen PEEP bei spontan atmenden Patienten nur mittels einer Ösophagusballonsonde verlässlich möglich ist, kommt in der Praxis eine Faustregel zur Anwendung, nach der bei Patienten mit COPD ein PEEP von 3 bis 5 mbar verwendet wird. Anlass für höhere PEEP-Werte sind:

- Adipositas

- Linksherzinsuffizienz

- Schlafassoziierte Erkrankungen

- begleitende schwere Oxygenierungsstörungen

Bei tief sedierten Patienten kann der PEEPi während eines expiratorischen Haltemanövers ermittelt und der PEEPe daran angepasst werden.

\section{Inspiratorischer Trigger}

Bei Patienten mit assistierter Beatmung sollte nach Möglichkeit ein Flow-gesteuerter Trigger von typischerweise 3-51/min zum Einsatz kommen. Ein zu empfindlicher Trigger begünstigt Auto-Triggern.

\section{Inspiratorische Anstiegssteilheit (Rampe)}

Aufgrund des hohen Atemantriebs und des Bedürfnisses nach einer langen Exspirationszeit wählt man bei Patienten mit COPD typischerweise einen steilen Druckanstieg von initial $200 \mathrm{~ms}$.

\section{Druckunterstützung und Tidalvolumen}

In den assistierten Modes entlastet die inspiratorische Druckunterstützung die Atemmuskulatur und stellt ein ausreichend hohes Tidalvolumen sicher. Mittels einer Druckunterstützung von 10-15 mbar kann bei druckunterstützter Beatmung typischerweise ein Tidalvolumen von $400-500 \mathrm{ml}$ erzielt werden. $\mathrm{Zu}$ hohe Tidalvolumina fördern hingegen die Überblähung und sollten daher vermieden werden. In den kontrollierten Modes gelten ähnliche Überlegungen.

\section{Atemfrequenz}

Im assistierten Mode ist eine Back-up-Atemfrequenz zu wählen, welche unter der Spontanatemfrequenz liegen sollte. Bei kontrollierter Beatmung verhindert eine niedrige Atemfrequenz (max. 10/min) die weitere Überblähung. 
Tab. 1 Interfaces bei NIV

\begin{tabular}{|c|c|c|}
\hline Interface & Einsatzmöglichkeit & Vorteil/Nachteil \\
\hline Nasenmaske: & $\begin{array}{l}\text { Stabiler Patient } \\
\text { Überwiegend außerklinisch }\end{array}$ & $\begin{array}{l}\text { +Sprechen, Essen, Trinken } \\
\text { +Abhusten } \\
\text { +weniger Komplikationen bei Erbrechen } \\
\text {-Mundleckagen }\end{array}$ \\
\hline Nasen-Mundmaske: & $\begin{array}{l}\text { Instabiler Patient } \\
\text { ICU/RCU } \\
\text { Außerklinisch } \\
\text { Präklinisch }\end{array}$ & $\begin{array}{l}\text { +weniger Leckagen } \\
\text { +hoher Beatmungsdruck möglich } \\
\text {-mehr Hautdefekte Auflagefläche } \\
\text {-Komplikationen bei Erbrechen }\end{array}$ \\
\hline Nasenoliven: & $\begin{array}{l}\text { Stabiler Patient } \\
\text { Überwiegend außerklinisch }\end{array}$ & $\begin{array}{l}\text { +freier Nasenrücken } \\
\text { +freies Gesichtsfeld } \\
\text {-Rhinitis } \\
\text {-hoher Beatmungsdruck nicht möglich }\end{array}$ \\
\hline Vollgesichtsmaske & $\begin{array}{l}\text { Instabiler Patient } \\
\text { ICU/RCU } \\
\text { Präklinisch }\end{array}$ & $\begin{array}{l}\text { +freier Nasenrücken } \\
\text { +hoher Beatmungsdruck möglich } \\
\text {-Augenirritationen } \\
\text {-Klaustrophobie } \\
\text {-Kondenswasser } \\
\text {-Komplikationen bei Erbrechen }\end{array}$ \\
\hline Individuell geformte Maske: & $\begin{array}{l}\text { Stabiler Patient } \\
\text { Überwiegend außerklinisch }\end{array}$ & $\begin{array}{l}\text { +optimale Passform } \\
\text { +weniger Hautdefekte } \\
\text {-teuer } \\
\text {-häufiger Ersatz notwendig } \\
\text {-stärkere Abnutzung } \\
\text {-Hygiene }\end{array}$ \\
\hline Helm & ICU/RCU & $\begin{array}{l}\text { +weniger Hautdefekte } \\
\text {-hoher Totraum } \\
\text {-geringe } \mathrm{CO}_{2} \text {-Clearance } \\
\text {-Triggerversagen }\end{array}$ \\
\hline
\end{tabular}

\section{Cycling}

Die Beendigung der Inspiration (Cycling) erfolgt bei assistierter Beatmung anhand des Abfalls des inspiratorischen Flows. Flow-Cycling bei $40 \%$ des inspiratorischen Spitzenflusses hat günstige Auswirkungen auf die dynamische Überblähung. Additiv kann auch die maximale Inspirationszeit limitiert werden (Time-Cycling), was insbesondere bei größeren Leckagen einen Vorteil bietet [3].

\section{Atemzeitverhältnis}

Zur Entblähung ist eine lange Expirationszeit erforderlich. Diese kann durch eine verhältnismäßig kurze Inspirationszeit (I:E Verhältnis) und/oder eine niedrige Atemfrequenz erzielt werden. Bei assistierten Modes sinkt die spontane Atemfrequenz des Patienten unter einer effektiven Abnahme der inspiratorischen Atemarbeit. In kontrollierten Modes wird typischerweise ein Atemzeitverhältnis von 1:3 bis 1:5 gewählt. Idealerweise geht der Flow am Ende der Exspiration gegen Null.

\section{Atemwegszugänge bei COPD}

Der häufigste und primär zu wählende Atemwegszugang bei COPD ist der nicht-invasive über ein geeignetes Interface. Tab. 1 gibt einen Überblick über die gängigsten Modelle mit den ihren Vor- und Nachteilen. Einige der Interfaces werden überwiegend außer- klinisch eingesetzt, andere sind nur intrahospital anzuwenden.

Sollte bei Versagen der NIV eine Intubation erforderlich sein, so ist wegen der pathophysiologisch erhöhten Resistance ein ausreichend dimensionierter Tubus zu wählen. HME-u. Bakterienfilter sowie Sprechaufsätze können den Atemwegswiderstand zusätzlich erhöhen und zu Airtrapping führen und sind nach Möglichkeit zu vermeiden.

\section{Präklinische Beatmung}

Die Anwendung und der Nutzen von invasiver und nicht-invasiver Beatmung bei akutem respiratorischen Versagen (Acute Respiratory Failure, ARF) und COPD sind gut untersucht und belegt [4].

Die Arbeiten basieren allerdings zum größten Teil auf Untersuchungen bei hospitalisierten Patienten. Für den präklinischen Bereich gibt es für die NIV bei Patienten im ARF wenig Evidenz [5-7].

Es bestehen aus pathophysiologischen Überlegungen keine Unterschiede in der Indikation für mechanische Ventilationsunterstützung zwischen präklinischen und hospitalisierten Patienten. Auch das primäre Ziel (Ersetzen/Unterstützen der Vitalfunktion Atmung) ist dasselbe. Das sekundäre Ziel (Wiederherstellen der eigenständigen Funktion der Atmung) steht aufgrund der kurzen Behandlungsdauer präklinisch nicht im Vordergrund [8, 9].

Unterschiede in der Art und Weise der Durchführung einer NIV ergeben sich durch die unterschiedlichen prä- und innerklinischen Ressourcen. Die Mög- 


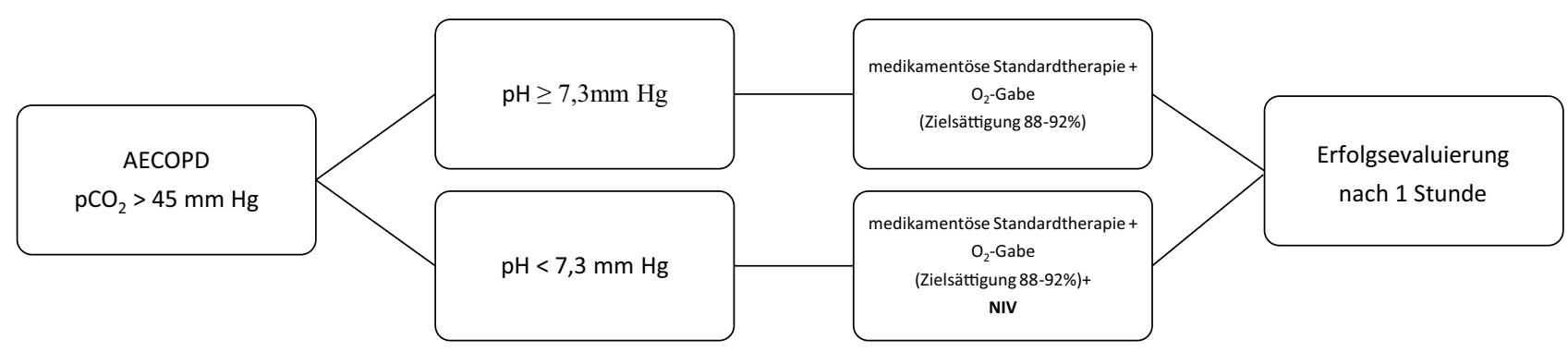

Abb. 2 Indikation für NIV bei AECOPD

lichkeit, zusätzlich zur klinischen Beurteilung auch die respiratorische Azidose als Entscheidungshilfe zur Einleitung einer NIV heranzuziehen fällt präklinisch mangels Verfügbarkeit einer Blutgasanalyse (BGA) meist weg, wäre aber sinnvoll. Dies betrifft in weiterer Folge auch die Erfolgskontrolle [8]. Für invasiv beatmete Patienten ist die Kapnographie ein erforderliches Monitoring-Tool (Tubuslage, Verlaufskontrolle). Das endtidale $\mathrm{CO}_{2}\left(\right.$ etCO $\left.\mathrm{CO}_{2}\right)$ ist bei kardiopulmonal kompromittierten Patienten allerdings als Surrogatparameter für die arterio-alveoläre $\mathrm{CO}_{2}$-Differenz $\left(\mathrm{AaDCO}_{2}\right)$ ungeeignet [10].

\section{Praktische Anwendung}

Turbinengeräte schneiden in Laborstudien hinsichtlich Triggergenauigkeit und Atemarbeit gemessen am Pressure-Time-Produkt (PTP) besser ab als Gasflowbetriebene Ventilatoren [11]. Der Nutzen hinsichtlich klinischem Effekt (Patienten-Ventilator-Synchronie) wurde bisher nicht untersucht. $\mathrm{Zu}$ fordern ist von modernen präklinischen Geräten zumindest eine Form der assistierten Beatmung und eine kontrollierte Beatmungsform mit adäquaten Möglichkeiten zur Alarmeinstellung. Eine Darstellung der Druck- und Flusskurve in ausreichender Qualität ist erforderlich, um Patienten-Respirator-Interaktionen zu beurteilen.

Das Outcome der NIV bei AECOPD hängt wesentlich vom Grad der Azidose und folglich einem frühzeitigen Therapiebeginn ab [12, 13]. Daraus ergibt sich die Forderung, NIV bereits während der präklinischen Phase zu beginnen. In einem modernen Notarztsystem müssen zusätzlich zu den technischen Voraussetzungen für die Durchführung von NIV auch die Erfahrung und die Ausbildung der Anwender garantiert sein.

\section{Nicht-invasive Beatmung bei akutem hyper- kapnischen Versagen im Rahmen einer COPD - Exazerbation (AECOPD)}

Die NIV ist beim akuten hyperkapnischen Versagen infolge einer COPD-Exazerbation gut etabliert, sie verringert nachweislich die Intubationsrate und verbessert das Überleben. Im Vergleich zur invasiven Beatmung weisen Patienten, die mit NIV behandelt werden können, eine geringere Rate an nosokomialen
Pneumonien auf, haben eine kürzere Verweildauer auf der Intensivstation und geringere Wiederaufnahmeraten. Ziele der NIV sind die Vermeidung einer Intubation und die Verringerung der Atemnot [14, 15].

\section{Patientenselektion und initiales Management}

Bei respiratorischer Azidose mit einem $\mathrm{pH}<7,3$ besteht unter Standardtherapie ein erhöhtes Intubationrisiko, es wird daher der unmittelbare Beginn einer NIV (Abb. 2) nach Ausschluss von Kontraindikationen (Tab. 2) empfohlen. Maßgeblich für die Indikationsstellung zur nicht-invasiven Akutbeatmung ist neben der Blutgasanalyse die ärztliche Beurteilung des klinischen Zustandes (Atemfrequenz, Atemmuster, Hämodynamik und Bewusstseinslage) des Patienten. Ein Monitoring ist für diese Patientengruppe unerlässlich. Neben den Routineparametern wie Sättigung, EKG und Blutdruck ist insbesondere auf PatientenVentilator-Interaktionen, auf die Atemfrequenz, sowie auf Leckagen zu achten.

\section{Respiratoreinstellung}

Meist wird eine assistierte Beatmung mit Druckunterstützung gewählt, aber auch assistiert/kontrollierte Beatmungsformen sind insbesondere bei $\mathrm{CO}_{2}$-bedingter Enzephalopathie mit zentralen Apnoen möglich.

\section{Versagen der nicht-invasiven Beatmung}

Der $\mathrm{pH}$-Wert ist der wichtigste prognostische Prädiktor von Patienten mit akut exazerbierter COPD. Ein fallender $\mathrm{pH}$ und eine progrediente Hyperkapnie, eine steigende Atemfrequenz, eine Verschlechterung der Oxygenierung, die neue hämodynamische Instabilität, Agitation oder Somnolenz, wie auch eine zunehmende Atemarbeit sind Zeichen eines Versagens der NIV. Dieses kann früh, aber auch verspätet, nach initialem Erfolg auftreten. Eine erste Kontrolle der Blutgasanalyse sollte ein bis zwei Stunden nach Beginn der NIV durchgeführt werden. Die häufigsten Ursachen für das Versagen von NIV bei COPD sind die Maskenintoleranz, unüberbrückbare Leckagen und Patienten-Ventilator-Asynchronie sowie unzureichendes Sekretmanagement. In Abhängigkeit von 
Tab. 2 NIV-Kontraindikationen

\begin{tabular}{l} 
Absolute Kontraindikationen für NIV: \\
Fehlende Spontanatmung, Schnappatmung \\
\hline Fixierte oder funktionelle Verlegung der Atemwege \\
Gastrointestinale Blutung oder lleus \\
\hline Nicht-COPD-bedingtes Koma \\
\hline
\end{tabular}

Relative Kontraindikationen für NIV:

Massive Agitation

Sekretverhalt trotz Bronchoskopie

Schwere Hypoxämie oder schwere Azidose $(\mathrm{pH}<7,1)$

COPD-bedingtes, hyperkapnisches Koma

Hämodynamische Instabilität

Anatomische $u / 0$ subjektive Interface-Inkompatibilität

Z. n. oberer gastrointestinaler $\mathrm{OP}$ der prognostischen Einschätzung und von dem Patientenwillen ist bei Versagen der NIV frühzeitig die Entscheidung zur Intubation oder die Therapiezieländerung mit begrenzenden oder deeskalierenden intensivmedizinischen Maßnahmen erforderlich. Eine Verzögerung der Intubation bei NIV-Versagen ist mit einer deutlich erhöhten Mortalität verbunden und daher zu vermeiden [16].

\section{Agitierter und unkooperativer Patient}

Primär sind die Aufklärung und adäquate Einstellung sowie die genaue Beobachtung der Wirkung und möglichen Probleme (Patient-Ventilator-Asynchronie) wesentlich. Bei ängstlichen Patienten kann es vorteilhaft sein, die Maske initial manuell durch das TherapieTeam anzupassen und noch nicht mit den Haltebändern zu fixieren, bis eine Toleranz gegeben ist [17]. Eine medikamentöse Unterstützung bei NIV-Intoleranz ist oft hilfreich, wobei ein möglichst wenig atemdepressiver Wirkstoff in adäquater Dosis gewählt werden sollte, z. B. Morphin und/oder Dexmedetomidin [18].

\section{Akute invasive Beatmung bei COPD}

\section{Klinischer Hintergrund}

Aufgrund der nachweislichen Vorteile der nicht-invasiven Beatmung (NIV) ist eine invasive Beatmung bei COPD die zweite Wahl. Bei absoluten Kontraindikationen oder Versagen der NIV kann jedoch eine invasive Beatmung über einen Endotrachealtubus erforderlich werden.

\section{Beatmungsformen}

Sofern mit dem Gasaustausch und der Atemmechanik vereinbar, sollten Patienten mit COPD innerhalb weniger Stunden nach der Intubation wach und kontaktierbar sein und bei erhaltenem Atemantrieb mittels eines assistierten Modus beatmet werden. Ein verbreiteter assistierter Mode ist die druckunterstützte Beatmung (PSV= pressure support ventilation $=\mathrm{ASB}^{\circledR}=$ assisted spontaneous breathing $\left.{ }^{\circledR}\right)$. Auch ein Volumen-gesteuerter Hub, welcher vom Patienten inspiratorisch getriggert wird, kann zur Anwendung kommen (Assist/Control Mode). Druck-gesteuerte assistierte Modes mit konstantem Tidalvolumen (z.B.
Volume support) „bestrafen“ potentiell Patienten mit hohem intrinsischem Atemantrieb durch eine niedrige Druckunterstützung und sind daher nicht erste Wahl.

Kommt bei ausgeschalteter Spontanatmung eine kontrollierte Beatmung zur Anwendung, so empfehlen sich druck- und zeitgesteuerte Modes. Bei hochgradiger Überblähung kann eine Steigerung der Beatmung die Rechtsherzfunktion zusätzlich verschlechtern ohne zur $\mathrm{CO}_{2}$-Clearance beizutragen. Lange Expirationszeiten oder kurze Diskonnektion mit manueller Entblähung sind hier hilfreich. Der Einsatz von extrakorporaler $\mathrm{CO}_{2}$-Eliminierung kann in diesen Fällen unterstützend wirken, wobei eine Prognoseverbesserung bisher nicht nachgewiesen wurde.

Die sogenannten Hybridmodi mit garantiertem Tidalvolumen verbinden die Vorteile der druckgesteuerten Beatmung (homogene Gasverteilung innerhalb der Lunge) mit den Vorteilen einer volumenkonstanten Beatmung [1]. Die Anwendung dieser Beatmungsformen erfordert ein engmaschiges klinisches Monitoring, da behebbare Ursachen einer respiratorischen Verschlechterung (z.B. Sekretobstruktion, Lungenödem oder Bronchospasmus) eventuell verschleiert und somit verspätet erkannt werden.

\section{Weaning bei COPD}

Weaning umfasst den Prozess der Übertragung der Atemarbeit eines beatmeten Patienten vom Respirator auf den Patienten selbst. Erfolgreiches Weaning wird definiert durch einen Zeitraum von $48 \mathrm{~h}$ ohne Notwendigkeit von Atemhilfen und ohne Zeichen der neuerlichen respiratorischen Erschöpfung [19].

Weaning-Patienten werden in drei Gruppen unterteilt (Tab. 3). Patienten mit prolongierter Entwöhnungsdauer weisen eine deutlich höhere Mortalität und Morbidität auf [20].

COPD-Patienten zeigen einen Trend zu schwierigem und prolongiertem Weaning [21, 22]. Dies ist wenig überraschend, da COPD-Patienten schon vor Einleiten einer Atemunterstützung alle Faktoren aufweisen, die ein Ungleichgewicht zwischen respiratorischer Last und Kapazität der Atempumpe bedingen [23].

$\mathrm{Zu}$ Beginn der Entwöhnung steht die Überprüfung, ob ein Patient den „Ready-to-Wean“ Kriterien 
Tab. 3 Weaningkategorien

\begin{tabular}{|c|c|c|c|c|}
\hline Gruppe & Kategorie & Definition & $\begin{array}{l}\text { Anteil an ICU- } \\
\text { Patienten (\%) }\end{array}$ & Mortalität (\%) \\
\hline 1 & Einfaches Weaning & $\begin{array}{l}\text { Erfolgreiches Weaning nach dem ersten Spontanatemversuch bzw. der ersten } \\
\text { Extubation }\end{array}$ & 59 & 13 \\
\hline 2 & Schwieriges Weaning & $\begin{array}{l}\text { Erfolgreiches Weaning nach inital erfolglosem Weaning, spätestens jedoch beim } \\
\text { 3. Spontanatemversuch innerhalb von } 7 \text { Tagen }\end{array}$ & 26 & 9 \\
\hline 3 & Prolongiertes Weaning & $\begin{array}{l}\text { Erfolgreiches Weaning nach mind. } 3 \text { erfolglosen Spontanatemversuchen oder } \\
\text { Beatmung länger als } 7 \text { Tage nach dem ersten Weaningversuch }\end{array}$ & 14 & 32 \\
\hline
\end{tabular}

Tab. 4 Readiness to Wean

\begin{tabular}{|c|c|}
\hline \multirow[t]{3}{*}{ Klinische Aspekte } & Ausreichende Hustenkraft \\
\hline & Ausreichendes Sekretmanagement \\
\hline & Ursächlicher Grund für die Intubation behoben \\
\hline \multicolumn{2}{|c|}{ Gemessene/objektive Parameter } \\
\hline Kardiale Situation & $\begin{array}{l}\text { Stabile kardiovaskuläre Situation (HF } \leq 140 / \mathrm{min}, \mathrm{RR}_{\text {sys }} 90-160 \mathrm{~mm} \mathrm{Hg} \text {, keine oder minimale } \\
\text { Dosis von Vasopressoren) } \\
\text { Stabile Metabolik }\end{array}$ \\
\hline $\begin{array}{l}\text { Oxygenierung/ } \\
\text { Beatmung }\end{array}$ & $\begin{array}{l}\mathrm{SaO}_{2}>90 \% \text { bei einem } \mathrm{FiO}_{2} \leq 0,4 \\
\text { PEEP } \leq 8 \mathrm{cmH}_{2} \mathrm{O}\end{array}$ \\
\hline $\begin{array}{l}\text { Ausreichende Lungen- } \\
\text { funktion }\end{array}$ & $\begin{array}{l}\mathrm{AF} \leq 35 / \mathrm{min} \\
\mathrm{max} . \mathrm{Pinsp} \leq 20-25 \mathrm{cmH}_{2} \mathrm{O} \\
\mathrm{VT}>5 \mathrm{ml} / \mathrm{kg} \\
\mathrm{VC}>10 \mathrm{ml} / \mathrm{kg} \\
\text { Keine signifikante respiratorische Azidose }\end{array}$ \\
\hline Sedierung/Neurologie & $\begin{array}{l}\text { Neurologisch stabiler Patient } \\
\text { Adäquate Reaktion }\end{array}$ \\
\hline \multicolumn{2}{|c|}{$\begin{array}{l}\text { HF Herzfrequenz, } R R_{s y s} \text { systolischer Blutdruck, } \mathrm{mm} \mathrm{Hg} \text { Millimeter Quecksilbersäule, } \mathrm{SaO}_{2} \text { arterielle Sauerstoffsätti- } \\
\text { gung, FiO }{ }_{2} \text { inspiratorische Sauerstofffraktion, } \mathrm{AF} \text { Atemfrequenz, } \mathrm{FiO}_{2} \text { maximaler inspiratorischer Beatmungsdruck, } \\
\mathrm{CmH}_{2} \mathrm{O} \text { Zentimeter Wassersäule, VT Atemzugvolumen, VC Vitalkapazität }\end{array}$} \\
\hline
\end{tabular}

entspricht (Tab. 4). Bei schwierigem oder prolongiertem Weaning wird bei COPD-Patienten zumeist eine diskontinuierliche Weaning-Strategie angewandt [24], die einem klassischen Trainingsmodell mit Phasen von intensiver Belastung und ausgedehnten Ruhepausen (=Beatmung) entspricht.

Alternativ besteht bei COPD-Patienten, die aufgrund eines hyperkapnischen akuten respiratorischen Versagens intubiert wurden auch die Möglichkeit, ein Weaning über NIV noch vor Erreichen der pulmonalen Kriterien zur „Readiness to Wean“ durchzuführen, ohne das Outcome zu verschlechtern [25, 26].

Für einen generellen prophylaktischen Einsatz der NIV nach konventionellem Weaning gibt es keine positiven Daten betreffend die Endpunkte Mortalität oder Re-Intubation, jedoch Empfehlungen bei Hochrisikopatienten, zu denen COPD-Patienten gezählt werden. [26, 27]. NIV bei neuerlichem respiratorischem Versagen nach Extubation (Postextubation Failure) zeigt keinen Vorteil [28, 29].

Der Wert von High-Flow Sauerstofftherapie über Nasenkanüle (High Flow Nasal Cannula - HFNC Oxygenation) ist noch unklar [30].

\section{Weaning-Protokolle}

Weaningprotokolle bieten im intensivmedizinischen Alltag eine Hilfestellung bei der Entwöhnung vom Respirator [24]. Es zeigt sich, dass bei einfachem oder schwierigem Weaning die Anwendung von Protokol- len die Dauer der mechanischen Beatmung unabhängig von der zugrundeliegenden Erkrankung verkürzen kann [31]. Bei prolongiertem Weaning profitieren insbesondere spezialisierte Abteilungen, die täglich ihre Patienten auf Entwöhnbarkeit hin untersuchen, nicht von weiterer protokollbasierter Unterstützung [32]. Frühe Reduktion der Sedierung und frühe Mobilisierung sind inzwischen grundsätzliche Prinzipen der Intensivmedizin [26]. Der Stellenwert der physiotherapeutischen Unterstützung im Weaning ist insbesondere bei dekonditionierten Patienten wie COPDKranken erwiesen [33].

\section{Beatmungsformen}

Der Spontanatemversuch (Spontaneous breathing trial - SBT) sollte bei COPD-Patienten initial mit milder Druckunterstützung, nicht ausschließlich mit CPAP oder T-Stück, vorgenommen werden. Im Allgemeinen gibt es keine Überlegenheit eines Beatmungsmodus für das Weaning, wobei SIMV nicht mehr als geeigneter Modus angesehen wird [19, 34]. Im Weaning ist eine engmaschige Kontrolle der atemphysiologischen Parameter zwingend, die Beatmung muss laufend an die Erfordernisse des Patienten angepasst werden. Auch hier gilt strikt, dass sowohl eine Überbeatmung als auch eine zu geringe Atemunterstützung zu vermeiden sind. Computerbasierte Weaning-Modes moderner Respiratoren werden bei schwerer COPD bisher nicht empfohlen [35, 36]. Der 
Tab. 5 Indikation zur auBerklinischen Beatmung bei COPD

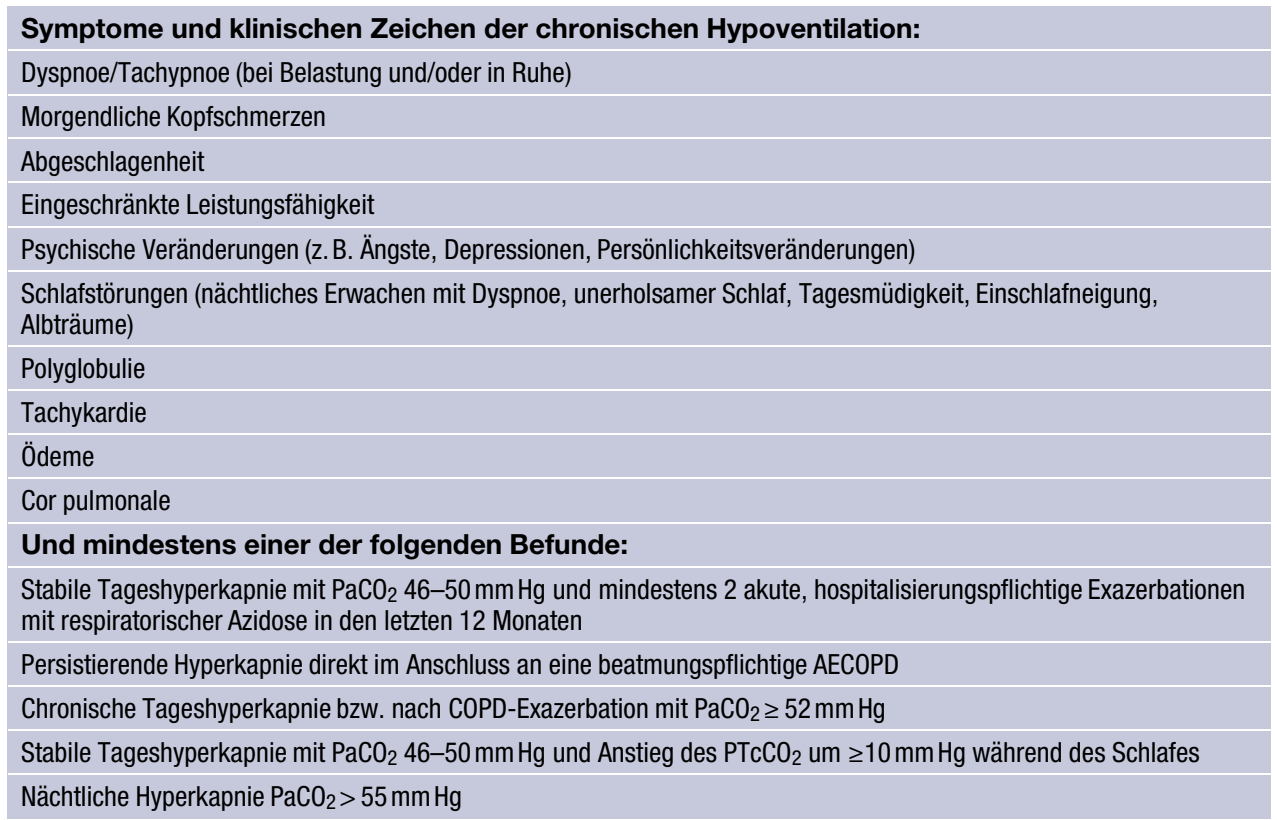

Einsatz extrakorporaler $\mathrm{CO}_{2}$-Eliminierung beim Weaning ist derzeit Gegenstand klinischer Studien [37].

\section{Tracheotomie}

Es gibt es keine Daten, die bei COPD-Patienten eine frühe vs. eine späte (nach dem 10. Beatmungstag) Tracheotomie favorisieren [38, 39]. Sollte das Weaning nur bis zur Kanüle gelingen, ist für eine Entlassung des Patienten mit Trachealkanüle jedenfalls ein epithelialisiertes Tracheostoma nötig [40]. Ein Weaning über NIV kann durch die Leckage nach Tracheostomaanlage erschwert sein.

\section{Weaning Failure}

Für die optimale Versorgung von COPD-Patienten, deren Entwöhnung prolongiert verläuft, empfiehlt es sich, eine spezialisierte Einrichtung frühzeitig $\mathrm{zu}$ kontaktieren. Zentren mit der Möglichkeit, die intensivmedizinische Beatmung stufenweise anzupassen (Step-Down-Units, Weaningzentren), verfügen über die Expertise, multimorbide und muskulär dekonditionierte Patienten adäquat $\mathrm{zu}$ versorgen. Intensivkapazitäten können so optimal genutzt beziehungsweise freigemacht werden. Insbesondere für nichtweanbare Patienten verfügen diese Einheiten über entsprechende Ausstattung und Erfahrung, um die Überleitung in die häusliche Umgebung oder eine Pflegeeinrichtung mit invasiver oder nicht-invasiver Beatmung erfolgreich durchzuführen. Auch die Entscheidung zur Therapiebegrenzung oder Palliation ist eine wesentliche Aufgabe im Weaning-Prozess [41, 42].

\section{Außerklinische Beatmung beim chronisch respiratorischen Versagen bei COPD}

War bisher beim akut respiratorischen Versagen bei AECOPD der Benefit einer nicht-invasiven Beatmung eindeutig in Studien belegt, so konnten erst in jüngerer Zeit Studien einen prognostischen Vorteil der NIV beim chronisch respiratorischen Versagen bei COPD zeigen [43]. Begründet wurde dies vor allem mit einer zuvor ineffektiven Beatmung hinsichtlich einer relevanten Reduktion der Hyperkapnie. Zusätzlich kann eine nicht-invasive Heimbeatmung zu einer Verbesserung der Lebensqualität und Symptome der chronisch ventilatorischen Insuffizienz führen. Trotz Hinweisen aus kleinen Studien ist der positive Effekt der nicht-invasiven Heimbeatmung auf die Exazerbationshäufigkeit und Hospitalisierungsrate noch unklar. Die Einstellung und Einschulung auf eine außerklinische Beatmung erfolgt in einem dafür spezialisierten Zentrum $[40,42]$.

Primär wird die außerklinische Beatmung nachts angewandt. Dabei sollte eine Beatmungsdauer von mehr als fünf Stunden erreicht werden. Im Krankheitsverlauf ist ein Ausweiten der Beatmung auf die Tageszeit möglich. Um die Mortalität zu senken ist eine suffiziente Beatmung erforderlich, das Ziel ist die Normokapnie bzw. eine relevante Reduktion des $\mathrm{pCO}_{2}$ von über $20 \%$ vom Ausgangswert.

\section{Indikation}

Kriterien für das Einstellen auf eine außerklinische Heimbeatmung sind die Kombination aus Symptomen/klinischen Zeichen der chronischen Hypoventilation und bestimmten Befundkonstellationen (siehe Tab. 5). 


\section{Beatmungszugang}

Als primärer Beatmungszugang sollte die NIV-Maske gewählt werden, Kriterien zur Auswahl des Interfaces sind in Tab. 1 dargestellt. Selten und zumeist als Folge eines Weaningversagens erfolgt das Einstellen von COPD-Patienten auf eine invasive Heimbeatmung. Beatmungszugang hierfür ist die Trachealkanüle über ein epithelialisiertes Tracheostoma.

\section{Respiratoreinstellung}

Aufgrund einer großen Herstelleranzahl besteht eine hohe Variabilität bei Respiratoren und den Beatmungsmodes. Wichtig ist, die Anwender der Beatmung auf den jeweiligen Respirator einzuschulen.

Eine spezielle Form der außerklinischen Beatmung bei COPD stellt die „high intensity NIV“ dar. Dabei werden typischerweise Druckwerte bis $30 \mathrm{cmH}_{2} \mathrm{O}$ erreicht und oft assistiert/druckkontrollierte Beatmungsmodi verwendet. Die Risiken von Überblähung, Volutrauma, Leckagen und Aerophagie sowie hämodynamische Nebenwirkungen stehen einer nicht gesicherten Verbesserung der Prognose gegenüber.

\section{Befeuchtung und Erwärmung}

Bei jeder Heimbeatmung ist die Verordnung einer Befeuchtung aus pathophysiologischen Gründen obligat. Verwendet werden sollte ein aktives Befeuchtungssystem (Verdampfungssysteme).

Die außerklinische Beatmung als notwendige therapeutische Maßnahme im Rahmen eines chronisch respiratorischen Versagens bei COPD führt zu einem Anspruch auf Behandlungssicherungspflege während der Beatmung. Die Bedienung und Überwachung des Beatmungsgerätes, bei invasiver Beatmung der Wechsel und die Pflege der Trachealkanüle und das Absaugen der oberen Luftwege, erfordern eine intensive häusliche Krankenpflege. Für Details bezüglich der Einstellung und Schulung auf eine außerklinische Beatmung verweisen wir auf den im Jahr 2016 veröffentlichten Konsensusreport der österreichischen Gesellschaft für Pneumologie [40].

\section{End of life - Palliation und Beatmung am Lebensende}

Das Ziel einer guten medizinischen Versorgung am Lebensende von COPD-Patienten ist in erster Linie die Beherrschung der Atemnot und der begleitenden Angst und Depression sowie der zunehmender Schwäche und der Kachexie [44]. Die End-StageCOPD wird klinisch definiert durch eine Atemflusseinschränkung (FEV1 $<30 \%$ predicted), einem schwer eingeschränkten und schlechter werdenden Performancestatus und eines der folgenden Kriterien: hohes Alter, Vorhandensein multipler Komorbiditäten, oder schwere Manifestationen/Komplikationen der
COPD [45, 46]. Bei COPD-Patienten im Endstadium und sich abzeichnendem Lebensende stellt sich die Frage nach dem Therapieziel (kurativ vs. palliativ), ob man eine Beatmungstherapie beginnt, von nichtinvasiver auf invasive Beatmung eskaliert oder einen Therapierückzug durchführt.

Für die Beantwortung dieser Fragen kann die Anwendung strukturierter ethischer Fragestellungen (inkl. Checklisten), Fallbesprechungen im Team und die Einholung einer externen Expertise, z.B. Ethikkonsil in der Entscheidungsfindung hilfreich sein und sollte schriftlich erfolgen [47-50].

Zur Symptombekämpfung sind nach Ausschöpfung der konservativen Therapiemöglichkeiten, inkl. der Langzeitsauerstoffgabe, Opioide das Mittel der ersten Wahl, wobei bereits niedrige Dosen $(2,5 \mathrm{mg}$ Morphin/4h) gut bei Atemnot wirken. Zusätzlich vermindert kühle Luft bzw. Luftzug in das Gesicht (mittels Ventilator) das Atemnotgefühl. Die Atmung kann durch physiotherapeutische Entblähung und Sekretmobilisation verbessert werden, ebenso wie durch die Aktivierung der muskulären Unterstützung, wie z. B. durch Mobilisierung und leichtes Muskeltraining. Zusätzlich vermindern Anxiolytika das subjektive Atemnotgefühl [44, 51]. Je nach Bedarf können sowohl Opioide, als auch Benzodiazepine, kontinuierlich mittels Perfusor verabreicht werden. Außerdem kann eine nicht-invasive Beatmung (als Ceilingtherapie) manchmal zur Verbesserung der Symptomatik und des Outcomes dieser Patienten beitragen [52].

Nach Jonson et al. [47] gilt es für eine medizinethische Entscheidung die Bereiche Indikation, Patientenwille, Lebensqualität und Kontextfaktoren zu berücksichtigen, wobei die Klärung des Therapiezieles (Indikation) und der damit als sinnvoll erachteten Behandlungsmaßnahme als vorrangig zu betrachten ist [48, 49, 53]. Die Indikation für eine medizinische Maßnahme ist gegeben, wenn damit das Therapieziel (z.B. Stabilisierung, Wiederherstellung, etc.) erreicht werden kann. Liegt die Erfolgsaussicht dafür aber unter $1 \%$ ist, so wird diese Maßnahme als sinnlos (futile) erachtet, wobei es da eine beachtliche Schwankungsbreite in der Auffassung gibt [54, 55]. Auch die Fortführung einer bereits begonnenen Maßnahme, die Ihr Ziel nicht mehr erreichen kann, wird als unethisch angesehen und soll konsequenterweise beendet werden [48, 49, 56].

Falls der Patient einsichts- und urteilsfähig ist, eine Vorausverfügung (Patientenverfügung oder Vorsorgevollmacht) bzw. Sachwalterschaft oder eingetragene Vertretungsbefugnis (Erwachsenenvertreter) besteht, wird der (mutmaßliche) Patientenwille als weiterer Entscheidungsfaktor berücksichtigt.

Prinzipiell sollten mit allen fortgeschrittenen und End-Stage-COPD-Patienten End-of-Life-Gespräche im Sinne eines Advanced Care Plannings (ACP) geführt werden, bei denen der prospektive Krankheitsverlauf und die Therapiemöglichkeiten und -belastungen besprochen werden, ebenso wie das Thema 
Vorsorgeverfügung und Gestaltung des letzten Lebensabschnittes [53, 57].

Die gesundheitsbezogene Lebensqualität als Kriterium für End-of-Life-Entscheidungen wird sehr unterschiedlich gesehen. Pearlmann und Jonsen [58] konnten bereits 1985 nachweisen, dass die Einschätzung einer schlechten Lebensqualität durch Ärzte mit einer niedrigeren Beatmungsrate einhergeht. Sprung et al. [59] zeigen in der ETHICATT Studie, dass für $88 \%$ der Ärzte die Lebensqualität bei End-of-LifeEntscheidungen wichtiger ist als das Leben selbst, dies aber nur bei $51 \%$ der Patienten und bei $63 \%$ der Angehörigen so ist. Die Mehrheit der Ärzte und Pflegenden aus dem Intensiv- und Palliativbereich sehen eine lebenserhaltende Maßnahme als sinnlos an, wenn sie keinen Benefit für die Lebensqualität bringt [53]. COPD Patienten lehnen bei einer schlecht empfundenen Lebensqualität aber nicht automatisch eine invasive oder nichtinvasive Beatmung ab [60]. Für sie muss die Belastung durch die Therapie durch die erreichbare Lebensqualität gerechtfertigt sein [48]. Von onkologischen Patienten weiß man, dass das Thema Lebensqualität gegen das Lebensende hin immer wichtiger wird [61]. Allerdings haben COPD-Patienten am Lebensende eine stärkere Symptombelastung über einen längeren Zeitraum als Karzinompatienten und bekommen weniger Palliativbetreuung [62, 63]. Wichtig ist, dass die zu erwartende Lebensqualität im Vorfeld kommuniziert wird [53].

Für ethische Entscheidungen am Lebensende soll nach Festlegung des Behandlungszieles geklärt werden, ob dieses erreichbar ist und wie stark die Belastung durch die Therapie ist. Falls die Indikation für die Therapie gegeben ist, steht die Frage nach dem Patientenwillen im Vordergrund, in die auch die zu erwartende Lebensqualität einfließt. Allfällige weitere interferierende Interessen sollten genannt und die Entscheidung verschriftlicht werden.

Funding Open access funding provided by Medical University of Vienna.

Interessenkonflikt E. Kink: Vortragshonorare von Dräger, Sanitas, Linde und Boehringer. W. Fritz: Vortragshonorare, Honorare für Advisory Boards oder Travel Grants von: Ratiopharm Teva, MEDahead, Mondial Congress, steirische Ärztekammer, Österreichische Gesellschaft für Pneumologie (ÖGP), Amgen, Astra Zeneca, ARGE steirische Lungenfachärzte, MedUniGraz, Boehringer Ingelheim, Linde, Genericon, GlaxoSmithKline Pharma, Habel, Menarini, Novartis, Vivisol. G.-C. Funk: Vortragshonorare, Honorare für Advisory Boards oder Travel Grants von: Actelion, Almirall, AstraZeneca, Bayer, BBraun, Boehringer Ingelheim, Dräger, Fresenius, GlaxoSmithKline Pharma, Gruenenthal, Heinen\&Löwenstein, Maquet, Menarini, Novartis, Nycomed, ORION Pharma, Pfizer, Roche Diagnostics, Takeda. O. Schindler: Interessenkonflikt der letzten 5 Jahre: Fellowship der ESICM, sponsored by ORION Pharma; Reisestipendien von ORION Pharma, Fresenius, Habel, Vivisol; Vortragshonorare und AdBoards: keine für dieses Paper relevanten. L. Erler, M. Gäbler, F. Krenn, G. Kühteubl und T. Wanke geben an, dass kein Interessenkonflikt besteht.
Open Access Dieser Artikel wird unter der Creative Commons Namensnennung 4.0 International Lizenz (http:// creativecommons.org/licenses/by/4.0/deed.de) veröffentlicht, welche die Nutzung, Vervielfältigung, Bearbeitung, Verbreitung und Wiedergabe in jeglichem Medium und Format erlaubt, sofern Sie den/die ursprünglichen Autor(en) und die Quelle ordnungsgemäß nennen, einen Link zur Creative Commons Lizenz beifügen und angeben, ob Änderungen vorgenommen wurden.

\section{Literatur}

1. Oczenski W. Atmen und Atemhilfen. 10. Aufl. Stuttgart: Thieme; 2017.

2. MacIntyre NR. Management of obstructive airway disease. In: Mechanical ventilation. 2. Aufl. Philadelphia, USA: ElsevierSaunders; 2009.

3. Chiumello D, et al. Effect of different cycling-off criteria and positive end-expiratory pressure during pressure support ventilation in patients with chronic obstructive pulmonary disease. Crit Care Med. 2007;35:2547-52.

4. Esteban A, Frutos-Vivar F, Muriel A, Ferguson ND, Penuelas $\mathrm{O}$, Abraira V, et al. Evolution of mortality over time in patients receiving mechanical ventilation. Am J Respir Crit Care Med.2013;188(2):128-30.

5. Bakke SA, Botker MT, Riddervold IS, Kirkegaard H, Christensen EF. Continuous positive airway pressure and noninvasive ventilation in prehospital treatment of patients with acute respiratory failure: a systematic review of controlled studies. Scand J Trauma Resusc Emerg Med. 2014;22(1):2156-2113.

6. Goodacre S, Stevens JW, Pandor A, Poku E, Ren S, Cantrell A, et al. Prehospital noninvasive ventilation for acute respiratory failure: systematic review, network meta-analysis, and individual patient data meta-analysis. Acad Emerg Med. 2014;21(9):960-70.

7. Mal S, McLeod S, Iansavichene A, Dukelow A, Lewell M. Effect of out-of-hospital noninvasive positive-pressure support ventilation in adult patients with severe respiratory distress: a systematic review and Meta-analysis. Annals of emergency medicine. Ann Emerg Med. 2014;63(5):600-1.

8. Lightowler JV, Wedzicha JA, Elliott MW, Ram FS. Noninvasive positive pressure ventilation to treat respiratory failure resulting from exacerbations of chronic obstructive pulmonary disease: Cochrane systematic review and metaanalysis. BMJ. 2003;326(7382):185-125.

9. Ozyilmaz E, Ugurlu AO, Nava S. Timing of noninvasive ventilation failure: causes, risk factors, and potential remedies. BMCPulm Med. 2014;14(1):19.

10. Prause G, Hetz H, LaudaP, Pojer H, Smolle-Juettner F, Smolle J. A comparison of the end-tidal-CO2 documented by capnometry and the arterial pCO2 in emergency patients. Resuscitation. 1997;35(2):145-8.

11. Thille AW, Lyazidi A, Richard J-CM, Galia F, Brochard L. A bench study of intensive-care-unit ventilators: new versus old and turbine-based versus compressed gas-based ventilators. Intensive Care Med. 2009;35(8):1368-76.

12. Ambrosino N, Vagheggini G. Non-invasive ventilation in exacerbations of COPD. COPD. 2007;2(4):471-6.

13. Dwarakanath A, Elliott MW. Noninvasive ventilation in the management of acute hypercapnic respiratory failure. Breathe. 2013;9(5):338-48.

14. Westhoff $\mathrm{M}$, et al. Nicht-invasive Beatmung als Therapie der akuten respiratorischen Insuffizienz; S3-Leitlinie Deutschen Gesellschaft für Pneumologie und Beatmungsmedizin. Pneumologie.2015;69:719-56. 
15. Rochwerg B, et al. Official ERS/ATS clinical practice guidelines: noninvasive ventilation for acute respiratory failure. EurRespirJ. 2017;50:1602426.

16. Simonds AK. ERS/practical handbook noninvasive ventilation. Eur Respir Soc. 2015. https://doi.org/10.1183/ 9781849840767.eph01

17. Davidson AC, Banham S, Elliott M, Kennedy D, Gelder C, Glossop A, et al. BTS/ICS guideline for the ventilatory management of acute hypercapnic respiratory failure in adults Thorax. Thorax. 2016;71:iil-ii35.

18. Senoglu N, Oksuz H, Dogan Z, Yildiz H, Demirkiran H, Ekerbicer $\mathrm{H}$. Sedation during noninvasive mechanical ventilation with dexmedetomidine or midazolam: a randomized, double-blind, prospective study. Curr Ther Res Clin Exp. 2010;71(3):141-53.

19. Boles J-M, Bion J, Connors A, Herridge M, Marsh B, Melot $\mathrm{C}$, et al. Weaning from mechanical ventilation. Eur Respir J. 2007;29(5):1033-56.

20. Funk G-C, Anders S, Breyer M-K, Burghuber OC, Edelmann $\mathrm{G}$, Heindl W, et al. Incidence and outcome of weaning from mechanical ventilation according to new categories. Eur RespirJ.2010;35(1):88-94.

21. Sellarés J, Ferrer M, Cano E, Loureiro H, Valencia M, Torres A. Predictors of prolonged weaning and survival during ventilator weaning in a respiratory ICU. Intensive Care Med. 2011;37(5):775-84.

22. Funk G-C, Bauer P, Burghuber OC, Fazekas A, Hartl S, Hochrieser $\mathrm{H}$, et al. Prevalence and prognosis of COPD in critically ill patients between 1998 and 2008. Eur Respir J. 2013;41(4):792-9.

23. Vassilakopoulos T, Zakynthinos S, Roussos C. Respiratory muscles and weaning failure. Eur Respir J. 1996;9(11):2383-400.

24. Schönhofer B, Geiseler J, Dellweg D, Moerer O, Barchfeld $\mathrm{T}$, Fuchs $\mathrm{H}$, et al. Prolongiertes weaning. Pneumologie. 2014;68(01):19-75.

25. Nava S, Ambrosino N, Clini E. Noninvasive mechanical ventilation in the weaning of patients with respiratory failure due to chronic obstructive pulmonary disease. Ann Intern Med. 1998;128(9):721-8.

26. Ouellette DR, Patel S, Girard TD, Morris PE, Schmidt GA, Truwit JD, et al. Liberation from me-chanical ventilation in critically ill adults: an official American College of chest Physicians/American Thoracic Society Clinical Practice guideline. Chest. 2017;151(1):166-80.

27. Vargas F, Clavel M, Sanchez-Verlan P, Garnier S, Boyer A, Bui $\mathrm{H}-\mathrm{N}$, et al. Intermittent noninvasive ventilation after extubation in patients with chronic respiratory disorders: a multicenter randomized controlled trial (VHYPER). Intensive Care Med.2017;9:1-11.

28. Ferrer M. Non-invasive ventilation in the weaning process. Minerva Anestesiol.2008;74(6):311-4.

29. Burns KE, Adhikari NKJ, Keenan SP, Meade M. Use of noninvasive ventilation to wean critically ill adults off invasive ventilation: meta-analysis and systematic review. Br Med J. 2009;338:b1574.

30. Papazian L, Corley A, Hess D, Fraser JF, Frat J-P, Guitton $\mathrm{C}$, et al. Use of high-flow nasal cannula oxygenation in ICU adults: a narrative review. Intensive Care Med. 2016;42(9):1336-49.

31. Ely EW, Baker AM, Dunagan DP, Burke HL, Smith AC, Kelly PT, et al. Effect on the duration of mechanical ventilation on identifying patients capable ofbreathing spontaneously. NEngl J Med. 1996;335(25):1864-9.

32. Blackwood B, Alderdice F, Burns KE, Cardwell C, Lavery G, O'Halloran P. Use of weaning protocols for reducing duration of mechanical ventilation in critically ill adult patients: Cochrane systematic review and meta-analysis. $\mathrm{Br}$ Med J.2011;342:c7237.

33. Schweickert WD, Pohlman MC, Pohlman AS, et al. Early physical and occupational therapy in mechanically ventilated, critically ill patients: a randomised controlled trial. Lancet. 2009;373:1874-82.

34. Hess D. Ventilator modes used in weaning. Chest. 2001;120(6):474S-6S.

35. El-Khatib MF, Bou-Khalil P. Clinical review: liberation from mechanical ventilation. Crit Care. 2008;12(4):221.

36. Lellouche F, Mancebo J, Jolliet P, Roeseler J, Schortgen F, Dojat M, et al. A multicenter randomized trial of computerdriven protocolized weaning from mechanical ventilation. Am J Respir Crit Care Med. 2006;174(8):894-900.

37. Morelli A, Sorbo L, Pesenti A, Ranieri VM, Fan E. Extracorporeal carbon dioxide removal (ECCO2R) in patients with acute respiratory failure. Intensive Care Med. 2017;28:1-12.

38. Hosokawa K, Nishimura M, Egi M, Vincent J-L. Timing of tracheotomy in ICU patients: a systematic review of randomized controlled trials. Crit Care. 2015;1:1-12.

39. Young D, Harrison DA, Cuthbertson BH, Rowan K. Effect of early vs late tracheostomy. JAMA. 2013;309(20):2121-9.

40. Schenk P, Eber E, Funk GC, Fritz W, Hartl S, Heininger P, Kink E, Kühteubl G, Oberwaldner B, Pachernigg U, Pfleger A, Schandl P, Schmidt I, Stein M. Non-invasive and invasive out of hospital ventilation in chronic respiratory failure : consensus report of the working group on ventilation and intensive care medicine of the Austrian Society of Pneumology. Wien Klin Wochenschr. 2016;128(Suppl 1):S1-S36.

41. Schneiderman LJ, Gilmer T, Teetzel HD, Dugan DO, Blustein J, Cranford R, et al. Effect of ethics consultations on nonbeneficial life-sustaining treatments in the intensive care setting: a randomized controlled trial. JAMA. 2003;290(9):1166-72.

42. Windisch W, Brambring J, Budweiser S, Dellweg D, Geiseler $\mathrm{J}$, Gerhard F, et al. Nichtinvasive und invasive Beatmung als Therapie der chronischen respiratorischen Insuffizienz S2Leitlinie der Deutschen Gesellschaft für Pneumologie und Beatmungsmedizin. Pneumologie. 2010;64:207-40.

43. Köhnlein T, Windisch W, Köhler D, Drabik A, Geiseler J, Hartl S, Karg O, Laier-Groeneveld G, Nava S, Schönhofer B, Schucher B, Wegscheider K, Criée CP, Welte T. Noninvasive positive pressure ventilation for the treatment of severe stable chronic obstructive pulmonary disease: a prospective, multicentre, randomised, controlled clinical trial.Lancet Respir Med.2014;2(9):698-705.

44. Barchfeld T. Palliativmedizin bei nichtmalignen chronisch pulmonalen Erkrankungen. Pneumologe. 2016;13:96-103.

45. Klimathianaki M, Mitrouska I, Georgopoulos D. Management of end-stage chronic obstructive pulmonary disease. In: Siafakas NM, Hrsg. Management of chronic obstructive pulmonary disease. 2006. S. 430-50.

46. Viegi G, Pistelli F, Sherrill DL, Maio S, Baldacci S, Carrozzi L. Definition, epidemiology and natural history of COPD. Eur Respir J. 2007;30:993-1013.

47. Jonsen AR, Siegler M, Winslade WJ. Clinical ethics: a practical approach to ethical decisions in clinical medicine. 8. Aufl. New York: McGraw-Hill; 2015.

48. Neitzke G, Burchardi H, Duttge G, Hartog C, Erchinger R, Gretenkort P, et al. Grenzen der Sinnhaftigkeit von Intensivmedizin - Positionspapier der Sektion Ethik der DIVI. Med Klin Intensivmed Notfmed. 2016; https://doi.org/10.1007/ s00063-016-0202-8.

49. Friesenecker B, Fruhwald S, Hasibeder W, Hörmann C, Hoffmann M, Krenn C, et al. Therapiezieländerungen auf der Intensivstation - Definitionen, Entscheidungsfindung 
und Dokumentation. Anasthesiol Intensivmed Notfallmed Schmerzther. 2016;48:216-23.

50. Valentin A, Druml W, Steltzer H, Wiedermann CJ. Recommendations on therapy limitation and therapy discontinuation in intensive care units: consensus Paper of the Austrian Associations of Intensive Care Medicine. Intensive Care Med.2008;34:771-6.

51. Ambrosino N, Simonds A. The clinical management in extremely severe COPD. Respir Med.2007;101:1613-24.

52. Creagh-Brown B, Shee C. Noninvasive ventilation as ceiling of therapy in end-stage chronic obstructive pulmonary disease. Chron Respir Dis. 2008;5:143-8.

53. Jox RJ, Schaider A, Marckmann G, Borasio GD. Medical futility at the end of life: the perspectives of intensive care and palliative care clinicians. J Med Ethics. 2012;38:540-5.

54. Gabbay E, Calvo-Broce J, Meyer KB, Trikalinos TA, Cohen J, KentDM. The empirical basis for determinations of medical futility. J Gen Intern Med. 2010;25:1083-9.

55. Schneiderman LJ, Jecker NS, Jonsen AR. Medical futility: response to critiques. Ann Intern Med. 1996;125:669-74.

56. ValentinA, DrumlW, SteltzerH. Konsensuspapier derIntensivmedizinischen Gesellschaften Österreichs: Empfehlungen zum Thema Therapiebegrenzung und-beendigung an Intensivstationen. Wien Klin Wochenschr. 2004;116:763-7.

57. Janssen DJA, Spruit MA, Schols JMGA, Wouters EFM. A call for high-quality advance care planning in outpati- ents with severe COPD or chronic heart failure. Chest. 2011;139:1081-8.

58. Pearlman RA, Jonsen A. The use of quality-of-life considerations in medical decision making. J Am Geriatr Soc. 1985;33:344-52.

59. Sprung CL, Carmel S, Sjokvist P, Baras M, Cohen SL, Maia P, et al. Attitudes of European physicians, nurses, patients, and families regarding end-of-life decisions: the ETHICATT study. Intensive Care Med. 2007;33:104-10.

60. Carlucci A, Guerrieri A, Nava S. Palliative care in COPD patients: is it only an end-of-life issue? Eur Respir Rev. 2012;21:347-54.

61. Winkler E, Heußner P. Vorausschauende Behandlungsplanung und Therapiebegrenzung. Dtsch Med Wochenschr. 2016;141:394-8.

62. Diaz-Lobato S, Smyth D, Curtis JR. Improving palliative care for patients with COPD. Eur Respir J. 2015;46:596-8.

63. Vermylen J, Szmuiowicz E, Kalhan R. Palliative care in COPD: an unmet area for quality improvement. Int J Chron ObstructPulmon Dis. 2015;10:1543-51.

Hinweis des Verlags Der Verlag bleibt in Hinblick auf geografische Zuordnungen und Gebietsbezeichnungen in veröffentlichten Karten und Institutsadressen neutral. 